\title{
Accounting for the Occlusion Effect with Insert Earphones
}

DOI: $10.3766 /$ jaaa. 17045

\author{
Dorothy Neave-DiToro* \\ Amanda DeSantolo ${ }^{\dagger}$ \\ Michael Bergen* \\ Adrienne Rubinstein*
}

\begin{abstract}
Background: There are clinical implications associated with knowing when the occlusion effect (OE) must be accounted for during bone conduction (BC) testing because spurious results can occur when errors are made in this regard. The amount of OE produced when insert earphones (IEs) are used varies in the literature; thus, further investigation is warranted.
\end{abstract}

Purpose: The purpose of this project was to determine the $\mathrm{OE}$ during $\mathrm{BC}$ threshold measurements under the following occluding conditions used clinically: when using partial insertion (PI) versus full insertion (FI) depth and when occluding one versus both ears.

Research Design: A descriptive within-subjects design was used in this study.

Study Sample: Twenty-two adults with mean four-frequency pure tone averages of $24 \mathrm{~dB} \mathrm{HL}$, aged 40-83 yr, participated.

Intervention: $\mathrm{BC}$ thresholds were obtained at 250,500 , and $1000 \mathrm{~Hz}$ under seven conditions: (1) both ears unoccluded, (2) left ear occluded with PI, (3) right ear occluded with PI, (4) both ears occluded with $\mathrm{PI}$, (5) left ear occluded with FI, (6) right ear occluded with $\mathrm{FI},(7)$ both ears occluded with FI. For PI, one half of the length of the IE was beyond the opening of the ear canal. For FI, the lateral edge of the foam insert was flush with the entrance to the ear canal.

Data Collection and Analysis: Mean OEs were compared with previously published data. In addition, variability in the data was examined using frequency distribution plots as well as cumulative frequency and percentile values.

Results: Mean OEs of $5-13 \mathrm{~dB}$ were present in all but the $\mathrm{FI}$ condition at $1000 \mathrm{~Hz}$ where the OE was $<3 \mathrm{~dB}$. Differences between $\mathrm{PI}$ and $\mathrm{FI}$ conditions were present at each frequency measured, irrespective of whether one or both ears were occluded. The shifts in threshold were consistently more prevalent and greater for the PI than the FI conditions overall. Mean differences between the one-ear and both-ears conditions were not clinically significant. Clinically significant variability in the data was noted, except when comparing the one-ear versus both-ears conditions.

Conclusions: Occluding the ear during initial BC measurements may artificially improve the thresholds and create or exaggerate an air-bone gap. Thus, initial BC testing should be performed unoccluded at 250, 500 , and $1000 \mathrm{~Hz}$. There is a need to account for the OE even when the IE is flush with the ear canal to avoid insufficient masking.

Key Words: air-bone gap, bone conduction, insert earphones, interaural attenuation, masking, occlusion effect, thresholds, unoccluded

Abbreviations: $\mathrm{BC}=$ bone conduction; $\mathrm{FI}=$ full insertion; IE = insert earphone; $\mathrm{OE}=$ occlusion effect; $\mathrm{PI}=$ partial insertion; SPL $=$ sound pressure level

*Department of Speech Communication Arts and Sciences, Brooklyn College, Brooklyn, NY; †Doctor of Audiology Program, The Graduate School of the City University of New York, New York, NY

Corresponding author: Dorothy Neave-DiToro, Department of Speech Communication Arts and Sciences, Brooklyn College, Brooklyn, NY 112102889; Email: dditoro@brooklyn.cuny.edu 


\section{ACCOUNTING FOR THE OCCLUSION EFFECT WITH INSERT EARPHONES}

$\mathrm{T}$ he presence of an occlusion effect (OE) during bone conduction (BC) testing was first reported by Wheatstone in 1827 (Goldstein and Hayes, 1965). The opening of the ear canal acts as a high-pass filter (Tonndorf, 1972); on occlusion, the low-frequency sound energy that would otherwise escape the canal is trapped to yield a higher in situ sound pressure level (SPL). The increased intensity of the signal results in the illusion of a more sensitive threshold. Several factors have been shown to affect the degree of the OE. For example, the inverse relationship between the $\mathrm{OE}$ and frequency is well-established, with the effect vanishing above $1000 \mathrm{~Hz}$ (Goldstein and Hayes, 1965; Edgerton and Klodd, 1977; Fagelson and Martin, 1998). Placement of the bone oscillator is a second factor; greater $\mathrm{OE}$ occurring with forehead placement of the bone oscillator as compared to mastoid placement has been reported by some (Edgerton and Klodd, 1977; Stenfelt and Reinfeldt, 2007), others have noted the opposite effect or no significant clinical difference (Goldstein and Hayes, 1965; Tonndorf, 1966; Fagelson and Martin, 1998). Placing the bone vibrator on the mastoid process is most commonly used in clinical settings today (Margolis and Stiepan, 2012).

A third factor affecting the $\mathrm{OE}$ is the selection of the transducer for masking, most commonly either supraaural or insert earphones (IE). Greater OEs consistently have been found for supra-aural headphones (Dean and Martin, 2000; Margolis and Moore, 2011). Khanna et al (1976) attributed this to the increased contact area with the head for supra-aural headphones compared with IEs. This phenomenon has been treated clinically as one of the advantages of using IEs during testing by increasing interaural attenuation for airconducted signals while reducing the OE. With regard to the degree of $\mathrm{OE}$ with IEs, mean values can vary across and within studies, depending on the protocol. For example, Dean and Martin (2000) found mean OEs of 17,14 , and $6 \mathrm{~dB}$ at frequencies of 250,500 , and $1000 \mathrm{~Hz}$, respectively. Margolis and Moore (2011), on the other hand, reported mean results of $3.3,4.2$, and $-0.8 \mathrm{~dB}$ at those frequencies. Anecdotal observation and reports suggest that some clinicians are not measuring or accounting for the $\mathrm{OE}$ when using IEs at any frequency, and furthermore, some are testing initial BC with IEs occluding both ears. If an OE does exist, the true bone-conduction results of a client could be misrepresented because the intensity increase in the nontest ear would not be taken into account. It should also be noted that recent investigation has yielded promising results for the use of circumaural headphones as an option that provides favorable results with regard to minimizing the OE (Margolis and Moore, 2011); however, as the goal of the current investigation was to examine procedures most relevant to current clinical practices, analysis of these transducers was not included.

Variation in the literature regarding the amount of $\mathrm{OE}$ reported with IEs may be attributed, in part, to insertion depth. They include depths of $7,10,11,13$, and $22 \mathrm{~mm}$, and are described with terms such as "shallow," "partial," "full," "intermediate," and "deep" insertion (Killion et al, 1988; Small and Stapells, 2003; Stenfelt and Reinfeldt, 2007; Stone et al, 2014). Other studies do not report the depth used (Fagelson and Martin, 1998; Guerrero-Aranda et al, 2016). It is generally agreed, however, that increasing insertion depth reduces the degree of OE (Zwislocki, 1953; Tonndorf, 1966; Killion et al, 1988). It has been reported that deep insertions, such as those entering the bony portion of the ear canal, might eliminate the OE (Tonndorf, 1966; Stenfelt and Goode, 2005). Clinically, however, even insertions not reaching the bony portion, although well into the canal, have been rejected because of issues of comfort in favor of partial insertion (PI) (Van Campen et al, 1990; Small and Stapells, 2003).

An additional issue of clinical relevance is whether occluding one ear versus both ears impacts the amount of $\mathrm{OE}$ measured; both methods anecdotally have been observed clinically. Studies regarding this issue have yielded variable results. Edgerton and Klodd (1977) found greater OEs with bilateral occlusion as compared to unilateral occlusion when using supra-aural headphones. Small and Stapells (2003) analyzed results for one versus both ears occluded using IEs and found significant differences in behavioral thresholds between the occlusion conditions at some frequencies but not at others. It is interesting to note the manner in which the data were collected for the one-ear occluded condition. In both studies, the choice of ear occluded was essentially random. It is therefore possible that the ear chosen for the one-ear occluded condition may not have been the ear which had the largest OE. Furthermore, for participants where there was greater bilateral occlusion, it may have been due to the ear with the smaller OE responding in the one-ear condition, and the ear with the larger OE responding in the both ears occluded condition. This also may be a factor in studies which only investigated one-ear occlusion. In some studies which examined the OE only in a one-ear occluded condition, masking was introduced to the nontest ear to assure that the test ear was the only ear being assessed for the OE (Stenfelt and Reinfeldt, 2007; Margolis and Moore, 2011). Whereas this method is successful in isolating the ear that is being examined, the potential existence of central masking, although small, cannot be ruled out. In any case, random assignment of one ear may 
underestimate the $\mathrm{OE}$ as the ear chosen may not represent the ear with the greater $\mathrm{OE}$.

Additional issues in the measurement of $\mathrm{OE}$ include potential experimenter bias, as well as ceiling effects. Ceiling effect here refers to the inability to accurately measure the $\mathrm{OE}$ of individuals who have occluded thresholds more sensitive than the minimal limits of clinical audiometers ( $-10 \mathrm{~dB}$ HL). Studies on this topic tend to include participants who had hearing sensitivity no poorer than $10 \mathrm{~dB}$ $\mathrm{HL}$ in some cases, and $20 \mathrm{~dB}$ HL in others (Edgerton and Klodd, 1977; Dean and Martin, 2000; Stenfelt and Reinfeldt, 2007; Margolis and Moore, 2011). The researchers failed to note, however, whether any of the occluded thresholds obtained were at $-10 \mathrm{~dB} \mathrm{HL}$, which could have resulted in an underestimation of the OE. In addition, with rare exception (Edgerton and Klodd, 1977), previous studies did not report whether the tester was blind regarding conditions while measuring behavioral thresholds.

Thus, because of the issues raised previously, the present study was designed to compare behavioral BC thresholds obtained unoccluded to those with different occluding conditions involving PI versus full insertion (FI) depth and one-ear versus both-ears occluded; the goal was to identify those conditions which make measurement of the OE clinically warranted when using IEs. In this investigation, thresholds were established psychoacoustically. OEs have also been measured using an acoustic method, defined as the difference in sound pressure level as measured with a probe microphone, with the ear occluded versus unoccluded (Tsai et al, 2005; Stone et al, 2014). Other studies have looked at both in an effort to make comparisons (Goldstein and Hayes, 1965; Fagelson and Martin, 1998; Stenfelt and Reinfeldt, 2007; Margolis and Moore, 2011; Reinfeldt et al, 2013). Goldstein and Hayes (1965) found that changes in SPL were greater than changes in threshold between unoccluded and occluded conditions. Fagelson and Martin (1998) reported that a strong association exists between ear canal SPL and behavioral measurements of the $\mathrm{OE}$; however, they did not find an exact decibel-for-decibel change between the measurements. Stenfelt and Reinfeldt (2007) as well as Margolis and Moore (2011) also reported relationships between these two types of measurements, both noting larger acoustic OEs than pyschoacoustic OEs. This is of importance as there may indeed be a significant OE occurring that can be measured objectively with a probe microphone, but which may not, however, be clinically relevant. Therefore, this investigation defined the OE solely in terms of changes in behavioral threshold.

\section{METHODS}

\section{Participants}

Twenty-two adults ( 7 males and 15 females) were recruited for participation, ranging in age from 40 to
83 yr. The selection criteria included (a) air conduction thresholds that were either within normal limits or showed no worse than a moderate sensorineural hearing loss (air conduction thresholds $\leq 55 \mathrm{~dB}$ HL and air-bone gaps $\leq 10 \mathrm{~dB}$ ), (b) symmetrical hearing as evidenced by no greater than a $15 \mathrm{~dB}$ difference in air conduction thresholds at 250, 500 and $1000 \mathrm{~Hz}$, (c) unremarkable otoscopic examination, (d) normal tympanograms (peak pressure $\pm 50 \mathrm{daPa}$ ), (e) present ipsilateral reflexes at 500, 1000, and $2000 \mathrm{~Hz}$, and (f) $-10 \mathrm{~dB} \mathrm{HL}$ responses at no more than two of the three test frequencies, 250, 500 and 1000 $\mathrm{Hz}$. This last criterion was included to limit the potential underestimation of the $\mathrm{OE}$. Mean four-frequency $(500,1000,2000$, and $4000 \mathrm{~Hz})$ pure tone averages were $24 \mathrm{~dB}$ HL. Approval for data collection was obtained from the Brooklyn College Institutional Review Board and informed consent was obtained from all participants. Participation was voluntary and unpaid.

\section{Procedures}

Testing was conducted in a double-walled test booth with a GSI AudioStar Pro audiometer, ER-3A IEs and a RadioEar B71 bone transducer calibrated according to ANSI S3.6-2010.

Air conduction thresholds were obtained at 250,500 , 1000,2000 , and $4000 \mathrm{~Hz}$ using pulsed tonal stimuli with IEs inserted flush with the ear canal. Either 13 or $10 \mathrm{~mm}$ foam insert tips were used depending on the size of the ear canal. A Weber test was performed with forehead placement of the bone oscillator at 250,500 , and $1000 \mathrm{~Hz}$ at $10 \mathrm{~dB}$ SL re: pure tone air conduction thresholds to determine the ear with the better cochlear response at each frequency because conductive hearing loss was ruled out. A method described by Stenfelt (2012) was used in which placement was determined according to the site of lateralization identified at a minimum of two out of three test frequencies. If this condition was not met because of midline identification, then the placement site was randomly chosen. If lateralization occurred at opposite locations for two frequencies, the oscillator was placed at the site of lateralization for the lowest frequency of the two.

$\mathrm{BC}$ thresholds were obtained at 250,500 , and $1000 \mathrm{~Hz}$ under seven conditions: (a) both ears unoccluded, (b) left ear occluded with PI, (c) right ear occluded with PI, (d) bilaterally occluded ears with PI, (e) left ear occluded with FI, (f) right ear occluded with FI, (g) bilaterally occluded ears with FI. For PI, one half of the length of the IE was beyond the opening of the ear canal. For FI placement, the outer edge of the foam insert was flush with the opening of the ear canal; this position also tended to be the point at which resistance was met, in terms of further inserting the tip into the ear (Clark and Roeser, 1988). To remove the potential for experimenter bias as well as an order effect, two experienced audiologists conducted the study in the 
following manner: for each participant, one of four randomizations of the test conditions was chosen to assure counterbalancing. One investigator set up the participant for each test condition and the other obtained thresholds without knowing the condition. The "blind" examiner was only aware of whether the frequency test order proceeded from 250 to $1000 \mathrm{~Hz}$, or the reverse order.

\section{RESULTS}

A lthough OEs were obtained for 22 participants from 250 to $1000 \mathrm{~Hz}$ for the PI and FI one-ear and both-ears conditions, the data from two participants (one male and one female) were not included in the analysis because of occluded ear responses at $-10 \mathrm{~dB}$ HL at all three test frequencies. Table 1 provides the means (and standard deviations) of the $\mathrm{OE}$, obtained by subtracting the occluded behavioral threshold from the unoccluded threshold at each frequency for each condition. As expected, the magnitude of the $\mathrm{OE}$ decreased with increasing frequency. Mean OEs of 5-13 dB were present in all but the FI condition at $1000 \mathrm{~Hz}$ where the $\mathrm{OE}$ was $<3 \mathrm{~dB}$. Differences between FI and PI conditions were present at each frequency measured, irrespective of whether one or both ears were occluded overall. The shifts in threshold were more prevalent for the PI than the FI conditions (see Tables $2-4)$. On the other hand, when comparing the data obtained for the one-ear versus both-ears conditions, mean results revealed less than a $1 \mathrm{~dB}$ difference between comparable conditions at each frequency for both PI and FI.

In addition to mean values, the variability in the data was also considered. Figures 1-3 represent frequency distribution plots showing the distribution of the $\mathrm{OE}$ measured across participants at each of the three test frequencies. PI versus FI and one-ear versus both-ears

Table 1. Mean OEs and Standard Deviations (in $\mathrm{dB}$ ) as a Function of PI vs. FI and One-Ear vs. Both-Ears

Occlusion

\begin{tabular}{clrrr}
\hline & & \multicolumn{3}{c}{ Frequencies } \\
\cline { 3 - 5 } & & $250 \mathrm{~Hz}$ & $500 \mathrm{~Hz}$ & $1000 \mathrm{~Hz}$ \\
\hline Partial insertion & & & & \\
One-ear occluded & Mean & 13.0 & 12.5 & 8.0 \\
& SD & 6.4 & 7.3 & 5.5 \\
Both-ears occluded & Mean & 12.8 & 13.3 & 8.5 \\
& SD & 7.2 & 7.1 & 5.4 \\
Full insertion & & & & \\
One-ear occluded & Mean & 9.8 & 7.0 & 2.8 \\
& SD & 7.5 & 6.2 & 7.2 \\
Both-ears occluded & Mean & 10.3 & 7.8 & 2.5 \\
& SD & 8.7 & 6.2 & 6.6 \\
\hline
\end{tabular}

Note: SD = standard deviation
Table 2. Cumulative Frequency (In Number of Participants) and Cumulative Percentile for Each Magnitude of OE for PI vs. FI and One-Ear vs. Both-Ears Occlusion at $250 \mathrm{~Hz}$

\begin{tabular}{|c|c|c|c|c|c|c|c|c|}
\hline \multirow[b]{2}{*}{ OE } & \multicolumn{2}{|c|}{ Partial One } & \multicolumn{2}{|c|}{ Partial Both } & \multicolumn{2}{|c|}{ Full One } & \multicolumn{2}{|c|}{ Full Both } \\
\hline & Freq. & $\%$ & Freq. & $\%$ & Freq. & $\%$ & Freq. & $\overline{\%}$ \\
\hline$-5 \mathrm{~dB}$ & 0 & 0 & 0 & 0 & 1 & 5 & 2 & $\overline{10}$ \\
\hline $0 \mathrm{~dB}$ & 1 & 5 & 2 & 10 & 4 & 20 & 5 & 25 \\
\hline $5 \mathrm{~dB}$ & 3 & 15 & 4 & 20 & 8 & 40 & 7 & 35 \\
\hline $10 \mathrm{~dB}$ & 10 & 50 & 10 & 50 & 11 & 55 & 9 & 45 \\
\hline $15 \mathrm{~dB}$ & 16 & 80 & 15 & 75 & 17 & 85 & 17 & 85 \\
\hline $20 \mathrm{~dB}$ & 18 & 90 & 18 & 90 & 20 & 100 & 19 & 95 \\
\hline $25 \mathrm{~dB}$ & 20 & 100 & 20 & 100 & 20 & 100 & 20 & 100 \\
\hline
\end{tabular}

occluded conditions are represented. In addition, numerals appearing above each bar represent the number of participants for whom OE may have been underestimated because of a ceiling effect resulting from the occluded threshold being audible at the lower limits of the audiometer ( $-10 \mathrm{~dB} \mathrm{HL})$. Results revealed $\mathrm{OE}$ values reaching $20-25 \mathrm{~dB}$ at 250 and $500 \mathrm{~Hz}$, as well as 15-20 dB at $1000 \mathrm{~Hz}$. These maxima occurred for PI as well as FI conditions, although the mean data for the PI condition were greater on average as previously noted. The number of participants whose OEs may have been affected by the audiometer limits was greatest at $250 \mathrm{~Hz}$ and in the partially occluded conditions. To obtain a fuller understanding of the data and its potential impact on clinical testing, the cumulative number of participants and cumulative percentiles for each degree of $\mathrm{OE}$ at each frequency and each condition were also calculated, as represented in Tables 2-4. Thus in Table 2 , for example, in the PI one-ear occluded condition at $250 \mathrm{~Hz}, 50 \%$ of the participants were shown to have up to, and including, an $\mathrm{OE}$ of $10 \mathrm{~dB}$, and $80 \%$ were shown to have up to, and including, a $15 \mathrm{~dB} \mathrm{OE}$.

Regarding the one versus both-ears conditions, the variability in the data were also explored. Results indicated there was either no difference or no more than a

Table 3. Cumulative Frequency (in Number of Participants) and Cumulative Percentile for Each Magnitude of OE for PI vs. FI and One-Ear vs. Both-Ears Occlusion at $500 \mathrm{~Hz}$

\begin{tabular}{|c|c|c|c|c|c|c|c|c|}
\hline \multirow[b]{2}{*}{ OE } & \multicolumn{2}{|c|}{ Partial One } & \multicolumn{2}{|c|}{ Partial Both } & \multicolumn{2}{|c|}{ Full One } & \multicolumn{2}{|c|}{ Full Both } \\
\hline & Freq. & $\%$ & Freq. & $\%$ & Freq. & $\%$ & Freq. & $\%$ \\
\hline$-5 \mathrm{~dB}$ & 0 & 0 & 0 & 0 & 1 & 5 & 0 & $\overline{0}$ \\
\hline $0 \mathrm{~dB}$ & 2 & 10 & 3 & 15 & 4 & 20 & 1 & 5 \\
\hline $5 \mathrm{~dB}$ & 6 & 30 & 4 & 20 & 11 & 55 & 3 & 15 \\
\hline $10 \mathrm{~dB}$ & 8 & 40 & 7 & 35 & 18 & 90 & 10 & 50 \\
\hline $15 \mathrm{~dB}$ & 16 & 80 & 13 & 65 & 18 & 90 & 16 & 80 \\
\hline $20 \mathrm{~dB}$ & 18 & 90 & 20 & 100 & 20 & 100 & 18 & 90 \\
\hline $25 \mathrm{~dB}$ & 20 & 100 & 20 & 100 & 20 & 100 & 20 & 100 \\
\hline
\end{tabular}


Table 4. Cumulative Frequency (in Number of Participants) and Cumulative Percentile for Each Magnitude of OE for PI vs. FI and One-Ear vs. Both-Ears Occlusion at $1000 \mathrm{~Hz}$

\begin{tabular}{|c|c|c|c|c|c|c|c|c|}
\hline \multirow[b]{2}{*}{$\mathrm{OE}$} & \multicolumn{2}{|c|}{ Partial One } & \multicolumn{2}{|c|}{ Partial Both } & \multicolumn{2}{|c|}{ Full One } & \multicolumn{2}{|c|}{ Full Both } \\
\hline & Freq. & $\%$ & Freq. & $\%$ & Freq. & $\%$ & Freq. & $\%$ \\
\hline$-5 \mathrm{~dB}$ & 0 & 0 & 1 & 5 & 5 & 25 & 5 & 25 \\
\hline $0 \mathrm{~dB}$ & 4 & 20 & 2 & 10 & 12 & 60 & 12 & 60 \\
\hline $5 \mathrm{~dB}$ & 9 & 45 & 8 & 40 & 15 & 75 & 15 & 75 \\
\hline $10 \mathrm{~dB}$ & 15 & 75 & 15 & 75 & 18 & 90 & 18 & 90 \\
\hline $15 \mathrm{~dB}$ & 20 & 100 & 20 & 100 & 19 & 95 & 20 & 100 \\
\hline $20 \mathrm{~dB}$ & 20 & 100 & 20 & 100 & 20 & 100 & 20 & 100 \\
\hline $25 \mathrm{~dB}$ & 20 & 100 & 20 & 100 & 20 & 100 & 20 & 100 \\
\hline
\end{tabular}

$5 \mathrm{~dB}$ difference in the amount of $\mathrm{OE}$ for every participant, except for a $10 \mathrm{~dB}$ difference at 250 or $500 \mathrm{~Hz}$ for four participants, and a $15 \mathrm{~dB}$ difference for one at $250 \mathrm{~Hz}$.

\section{DISCUSSION}

$\mathrm{T}$ he purpose of the current study was to determine whether unoccluded BC thresholds significantly differed clinically (i.e., $\geq 5 \mathrm{~dB}$ shift) in comparison with a number of relevant occluded conditions. Identifying an occluded condition which does not result in a clinically significant $\mathrm{OE}$ would validate a protocol that would potentially reduce test time by allowing the clinician to test initial BC as well as perform masking procedures when necessary without physically changing test set up. At the same time, it could avoid artificially improving the $\mathrm{BC}$ thresholds which would otherwise create or exaggerate an air-bone gap. One clinically desirable occluded condition involves the use of PI because of the greater reported comfort of this approach (Small and Stapells, 2003; Margolis and Moore, 2011). The results of the present investigation indicated that OEs were larger and more prevalent with PI than with FI at all frequencies tested. Overall the mean OEs measured in the present study were similar to those reported by Dean and Martin (2000) who used a similar protocol to the one-ear occluded condition with their mastoid placement and IEs. Their mean OEs of 16,10 and $6 \mathrm{~dB}$ at 250, 500, and $1000 \mathrm{~Hz}$, respectively with PI are within $3 \mathrm{~dB}$ of our data. Thus, the results of the present investigation corroborated their results; taken together, these findings support the need to account for the $\mathrm{OE}$ with PI when using IEs.

Although potentially less comfortable, FI of IEs would be desirable if it avoided the need to account for the OE. In the one-ear condition, our mean results were within 1-2 dB of those of Dean and Martin (2000) who obtained mean OEs of 9, 6, and $1 \mathrm{~dB}$ at 250, 500, and $1000 \mathrm{~Hz}$, respectively. Based on their findings, Dean and Martin (2000) concluded that FI is preferable: in addition to establishing that lower initial masking levels would be required, they concluded that accounting for the $\mathrm{OE}$ would only be needed at 250 and $500 \mathrm{~Hz}$. Before ruling out the need to account for the OE, however, the variability in the data should be considered. As shown in Figure 3, there is dispersion in the data even at the $1000 \mathrm{~Hz}$ FI condition. In fact, of the 20 participants, there were those who demonstrated OEs of $15 \mathrm{~dB}$ or more for every condition tested. Thus, choosing to assume the absence of an $\mathrm{OE}$, even in the FI condition, does not appear to be prudent. In fact, PI is often preferred clinically (Laws et al, 1993; Small and Stapells, 2003; Margolis and Moore, 2011). In the current study, we were able to reach an insertion flush with the ear canal in all participants by using a smaller IE in two cases. Therefore, given the advantages of FI, we recommend that PI should be used only after FI is first attempted and not successful.

Clinical guidelines for addressing the OE recommend measurement for each client individually (Silman and Silverman, 1991; Gelfand, 2007; Yacullo, 2009) or use of fixed values based on the literature (Yacullo, 2009). The commonly proposed fixed values tend to be consistent with the data of Dean and Martin (2000), that is, $10 \mathrm{~dB}$ at 250 and $500 \mathrm{~Hz}$. It is useful to assess these recommendations within the context of the cumulative percentiles provided in Tables 2-4. At 250 $\mathrm{Hz}$, in the one-ear FI condition, [which is most similar to the protocol of Dean and Martin (2000)], accounting for up to an $\mathrm{OE}$ of $10 \mathrm{~dB}$ would be appropriate for only $55 \%$ of the participants. Assuming no $\mathrm{OE}$ at $1000 \mathrm{~Hz}$ would only account for $60 \%$ of the participants. Using these same guidelines with partially occluded ears would account for the OE in even fewer participants. Comparing the recommendations to the both-ears occluded condition would result in similar findings. In the case of 500 $\mathrm{Hz}$ in the one-ear FI condition, the $10 \mathrm{~dB}$ criterion surprisingly did account for the $\mathrm{OE}$ in most of the participants $(90 \%)$, unlike the other frequencies and other conditions at $500 \mathrm{~Hz}$.

Thus, aside from the more atypical results at $500 \mathrm{~Hz}$ in one condition, all findings using the fixed values were predictable. About half of those tested would have OEs that were larger, and thus the amount of masking needed would be potentially insufficient. This would be comparable with deciding whether masking for air conduction is needed, and then basing the decision on average interaural attenuation rather than minimum interaural attenuation values. We are in agreement with the recommendations of Studebaker (1967) to use maximum values for the $\mathrm{OE}$ as well as minimum values for interaural attenuation to avoid the potential risk of undermasking. Thus, if choosing to use a fixed value in accounting for the $\mathrm{OE}$, 

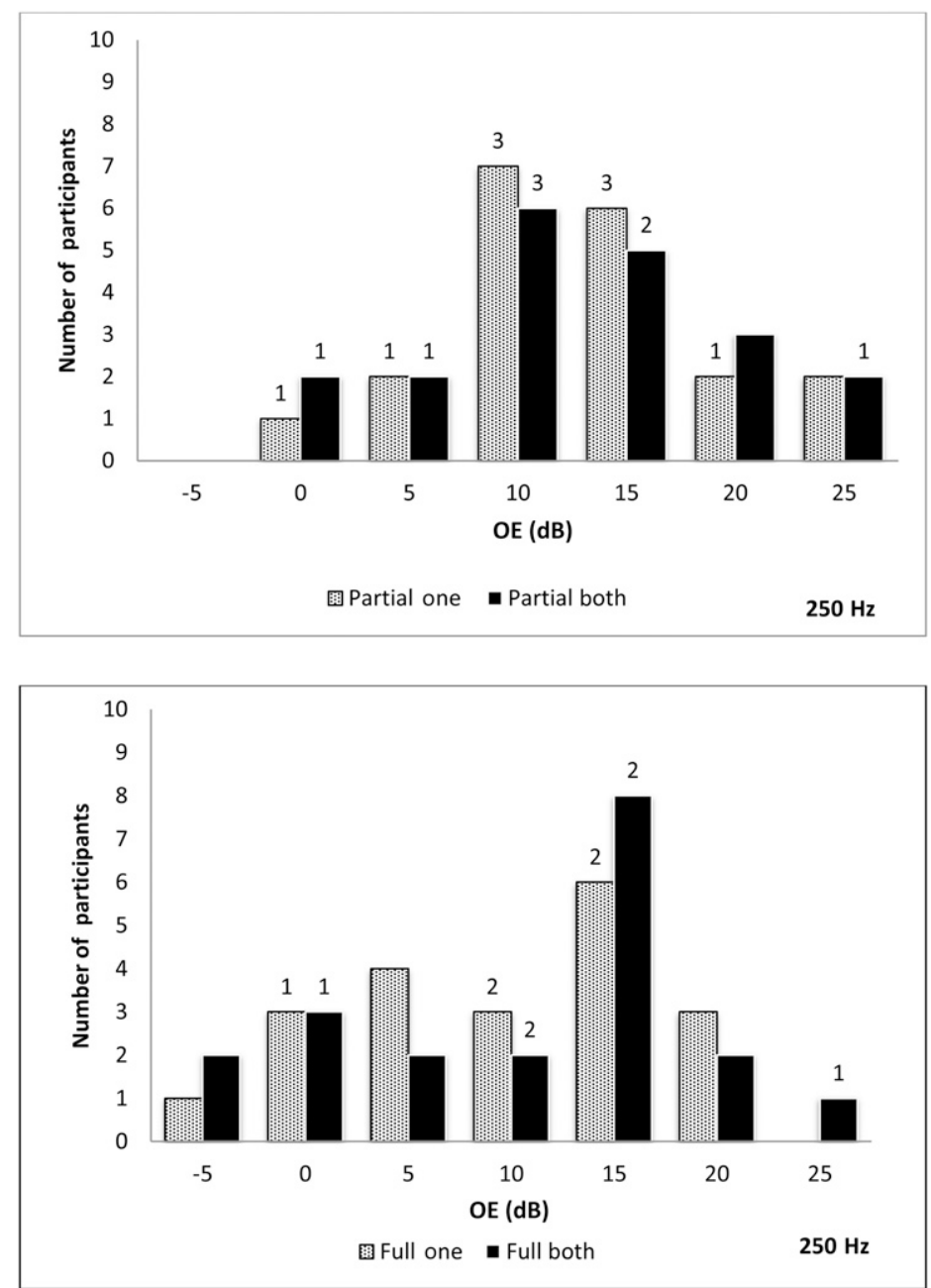

Figure 1. Frequency distribution (in number of participants) as a function of OE magnitude (in $\mathrm{dB}$ ) at $250 \mathrm{~Hz}$ in the one-ear and bothears conditions. Data using PI are in the top graph and FI data are in the bottom graph. Numerals over select bars represent the number of participants whose OE may have been underestimated because of a ceiling effect.

the percentiles provided in Tables $2-4$ could be used as a guide. In choosing a value, however, one should keep in mind that these data may underestimate the maximum OE because (a) they are based on only 20 participants and, (b) ceiling effects were noted in some cases. The issue of potential ceiling effects in participants with normal hearing was not addressed in previous studies, as noted earlier. It is recommended that further investigations in this area should include only participants who have no ceiling effects at all in the occluded condition, such as those with no better than -5 $\mathrm{dB}$ HL occluded thresholds. In place of using fixed values for the $\mathrm{OE}$, determination of an individual's own $\mathrm{OE}$ has been recommended instead by some (Silman and Silverman, 1991; Gelfand, 2007). Although marginally more time-consuming, an advantage of this method is that only the necessary amount of masking will be used. Ceiling effects may be encountered when attempting to determine an individual's $\mathrm{OE}$ as well, in which case having fixed values may be helpful here also.
Interestingly, mean results for occluding one-ear versus both-ears did not make a clinically significant difference, thus suggesting that there is not generally a cumulative effect between the ears. It is rather the ear with the greatest amount of $\mathrm{OE}$ that is responding in a "both-ears occluded" condition. The current results differ from previous investigations (Edgerton and Klodd, 1977; Small and Stapells, 2003) which found differences in the degree of OE depending on whether one or both of the ears were occluded. It may be recalled that a main difference in our study compared with previous research was in the testing of both right and left one-ear conditions under the hypothesis that the bilateral condition would compare more similarly with the ear with the greatest $\mathrm{OE}$, which we found to be the case. The homogeneous results found for these two conditions would suggest that, following testing in the unoccluded condition, one could consider placing IEs in both ears simultaneously. One would thus be obtaining and using the larger $\mathrm{OE}$. 

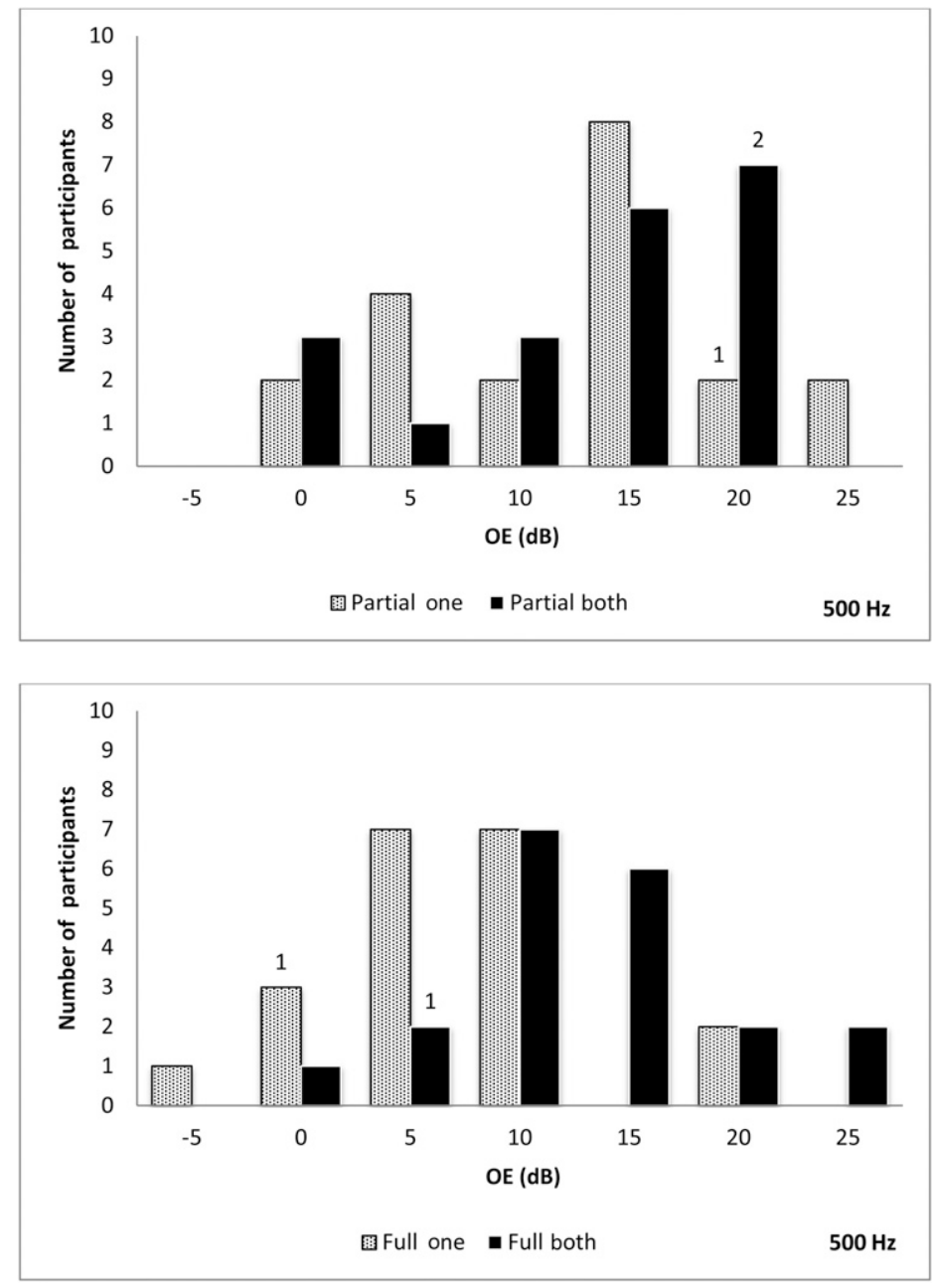

Figure 2. Frequency distribution (in number of participants) as a function of $\mathrm{OE}$ magnitude (in $\mathrm{dB}$ ) at $500 \mathrm{~Hz}$ in the one-ear and bothears conditions. Data using PI are in the top graph and FI data are in the bottom graph. Numerals over select bars represent the number of participants whose $\mathrm{OE}$ may have been underestimated because of a ceiling effect.

\section{CONCLUSIONS}

- Initial BC testing should be performed unoccluded at 250,500 , and $1000 \mathrm{~Hz}$. In addition to the need to account for the OE even with FI to avoid insufficient masking, occluding the ear during initial measurements may artificially improve the BC thresholds and create or exaggerate an air-bone gap.

- If choosing to use fixed values, we recommend basing the decision on the data from Tables 2-4, using the correction specific to the depth of insertion used. Using a value for the $\mathrm{OE}$ that is $5 \mathrm{~dB}$ larger than the one that accounts for all the participants in the current study does not appear to be unduly conservative because these data are based on only 20 participants.

- Calculating an individual's own OE is advantageous because no more than the necessary amount of masking will be used. The following procedure is recommended for measuring an individual's $\mathrm{OE}$ :
- Obtain initial BC thresholds with unoccluded ears.

- Place IE in canal until at least the outer edge is flush with the opening of the ear canal.

- Reassess the BC thresholds with the IE in the ear without masking at 250,500, and $1000 \mathrm{~Hz}$.

- Subtract the BC threshold measured with the occluded ear from the initial BC responses to obtain the $\mathrm{OE}$ for that individual at each frequency.

- For each frequency and each ear, add the amount of $\mathrm{OE}$ measured at that frequency to the starting level of the masking in the nontest ear.

- If masking will be needed in each ear, the IE should not be placed in both ears simultaneously, as this may result in an $\mathrm{OE}$ in the TE and a false $\mathrm{ABG}$, in some cases.

- Because of the potential underestimation of OE when using participants with normal hearing, further assessment of fixed values to account for the OE should 

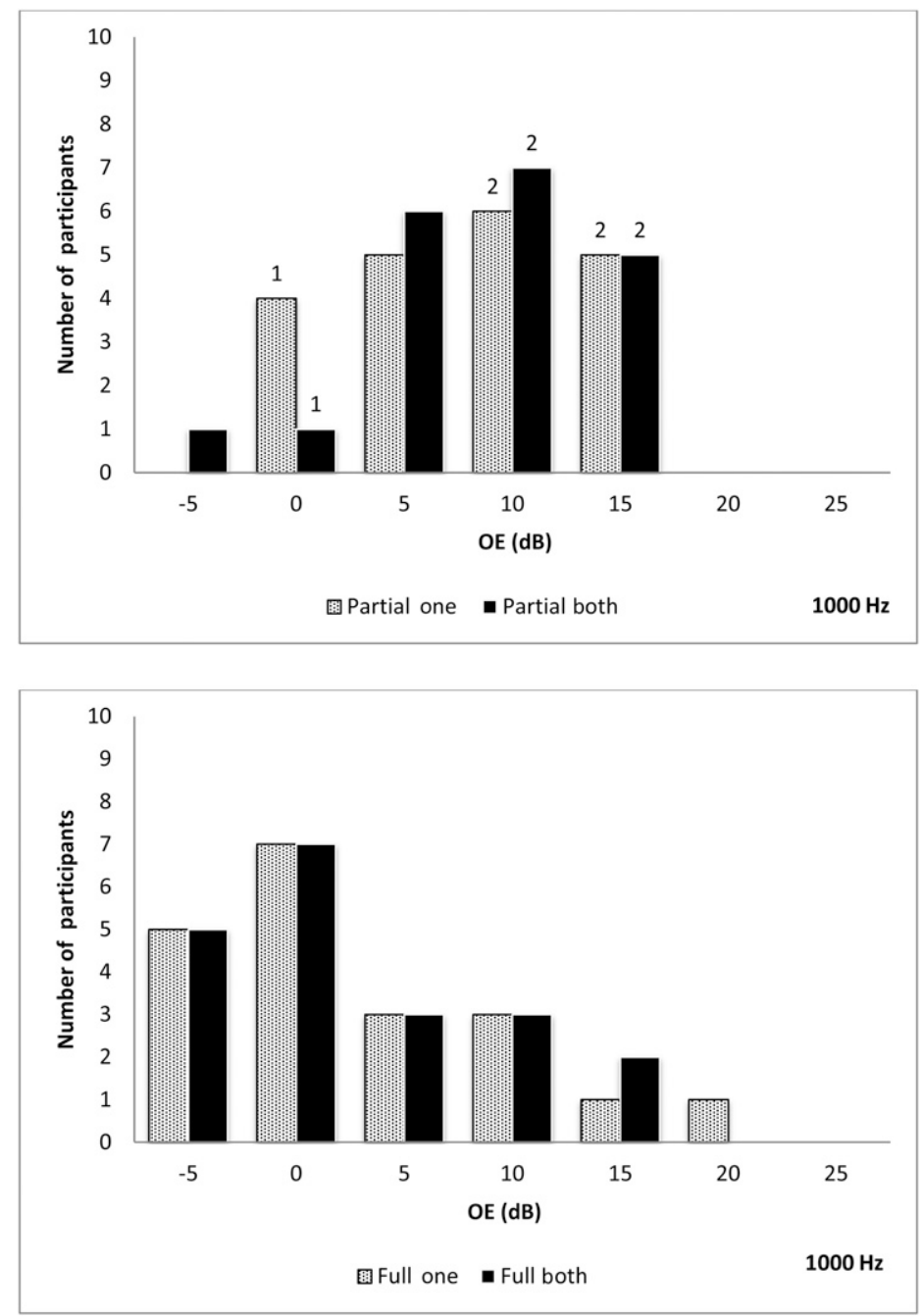

Figure 3. Frequency distribution (in number of participants) as a function of OE magnitude (in $\mathrm{dB}$ ) at $1000 \mathrm{~Hz}$ in the one-ear and bothears conditions. Data using PI are in the top graph and FI data are in the bottom graph. Numerals over select bars represent the number of participants whose OE may have been underestimated because of a ceiling effect.

include participants whose thresholds do not reach the minimum limits of the audiometer $(-10 \mathrm{~dB} \mathrm{HL})$.

Acknowledgments. A capstone research project, which served as a pilot study for this investigation, was submitted by Amanda DeSantolo to the Graduate Faculty in Audiology in partial fulfillment of the requirements for the degree of Doctor of Audiology, The City University of New York. The authors would like to acknowledge Arlene Neuman, Ida Kukliansky, and Merav Ben-David for their input in the preparation of the article. They also thank the peer reviewers for their suggested revisions and comments on a previous draft of this paper.

\section{REFERENCES}

Clark JL, Roeser RJ. (1988) Three studies comparing performance of the ER-3A tubephone with the TDH-50P earphone. Ear Hear 9(5):268-274.
Dean MS, Martin FN. (2000) Insert earphone depth and the occlusion effect. Am J Audiol 9(2):131-134.

Edgerton BJ, Klodd DA. (1977) Occlusion effect in bone conduction pure tone and speech audiometry. J Am Audiol Soc 2(4):151-158.

Fagelson MA, Martin FN. (1998) The occlusion effect and ear canal sound pressure level. Am J Audiol 7(2):50-54.

Gelfand SA. (2007) Essentials of audiology. New York: Thieme.

Goldstein DP, Hayes CS. (1965) The occlusion effect in bone conduction hearing. J Speech Hear Res 8(2):137-148.

Guerrero-Aranda A, Mijares-Nodarse E, Hernandez-Perez H, Torres-Fortuny A. (2016) Confirmation of previous results of the occlusion effect through auditory steady-state responses in normal-hearing adults. Indian J Otol 22(4):231-236.

Khanna SM, Tonndorf J, Queller JE. (1976) Mechanical parameters of hearing by bone conduction. J Acoust Soc Am 60(1):139-154.

Killion MC, Wilber LA, Gundmundson GI. (1985) Insert earphones for more interaural attenuation. Hear Instr 36(2):34. 
Killion MC, Wilber LA, Gudmundsen GI. (1988) Zwislocki was right. Hear Instr 39(1):14-18.

Laws DW, Roller SM, Perry CK. (1993) Interaural attenuation of a click stimulus using deep and partial placement of an insert earphone. Am J Audiol 2(3):60-63.

Margolis RH, Moore BC. (2011) AMTAS(®): automated method for testing auditory sensitivity: III. Sensorineural hearing loss and air-bone gaps. Int J Audiol 50(7):440-447.

Margolis RH, Stiepan SM. (2012) Acoustic method for calibration of audiometric bone vibrators. J Acoust Soc Am 131(2): $1221-1225$.

Reinfeldt S, Stenfelt S, Håkansson B. (2013) Estimation of bone conduction skull transmission by hearing thresholds and earcanal sound pressure. Hear Res 299:19-28.

Silman S, Silverman CA. (1991) Auditory diagnosis: principles and applications. Academic Press.

Small SA, Stapells DR. (2003) Normal brief-tone bone-conduction behavioral thresholds using the B-71 transducer: three occlusion conditions. J Am Acad Audiol 14(10):556-562.

Stenfelt S. (2012) Transcranial attenuation of bone-conducted sound when stimulation is at the mastoid and at the bone conduction hearing aid position. Otol Neurotol 33(2):105-114.
Stenfelt S, Goode RL. (2005) Bone-conducted sound: physiological and clinical aspects. Otol Neurotol 26(6):1245-1261.

Stenfelt S, Reinfeldt S. (2007) A model of the occlusion effect with bone-conducted stimulation. Int J Audiol 46(10):595-608.

Stone MA, Paul AM, Axon P, Moore BC. (2014) A technique for estimating the occlusion effect for frequencies below $125 \mathrm{~Hz}$. Ear Hear 35(1):49-55.

Studebaker GA. (1967) Clinical masking of the nontest ear. J Speech Hear Disord 32(4):360-371.

Tonndorf J. (1972) Foundations of Modern Auditory Theory. II.

Tsai V, Ostroff J, Korman M, Chen JM. (2005) Bone-conduction hearing and the occlusion effect in otosclerosis and normal controls. Otol Neurotol 26(6):1138-1142.

Van Campen LE, Sammeth CA, Peek BF. (1990) Interaural attenuation using etymotic ER-3A insert earphones in auditory brain stem response testing. Ear Hear 11(1):66-69.

Yacullo WS. (2009) Clinical masking. In: Katz J, Medwetsky L, Burkard R, Hood L, eds. Handbook of Clinical Audiology. 6th ed. Philadelphia, PA: Lippincott, Williams \& Wilkins, 80-115.

Zwislocki J. (1953) Acoustic attenuation between the ears. J Acoust Soc Am 25(4):752-759. 